\title{
Robert le Diable, Édition bilingue, Publication, traduction, présentation et notes par Élisabeth Gaucher
}

\author{
Maria Colombo Timelli
}

\section{(2) OpenEdition \\ 1 Journals}

\section{Édition électronique}

URL : http://journals.openedition.org/studifrancesi/9753

DOI : ERREUR PDO dans /localdata/www-bin/Core/Core/Db/Db.class.php L.34 : SQLSTATE[HY000]

[2006] MySQL server has gone away

ISSN : 2427-5856

\section{Éditeur}

Rosenberg \& Sellier

\section{Édition imprimée}

Date de publication : 1 octobre 2007

Pagination : 412-414

ISSN : 0039-2944

\section{Référence électronique}

Maria Colombo Timelli, « Robert le Diable, Édition bilingue, Publication, traduction, présentation et notes par Élisabeth Gaucher », Studi Francesi [En ligne], 152 (LI | II) | 2007, mis en ligne le 30 novembre 2015, consulté le 07 janvier 2021. URL : http://journals.openedition.org/studifrancesi/9753; DOI : https://doi.org/10.4000/studifrancesi.9753

Ce document a été généré automatiquement le 7 janvier 2021.

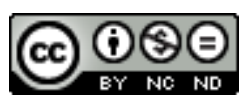

Studi Francesi è distribuita con Licenza Creative Commons Attribuzione - Non commerciale - Non opere derivate 4.0 Internazionale. 


\title{
Robert le Diable, Édition bilingue, Publication, traduction, présentation et notes par Élisabeth Gaucher
}

\author{
Maria Colombo Timelli
}

\section{RÉFÉRENCE}

Robert le Diable, Édition bilingue, Publication, traduction, présentation et notes par ÉLISABETH GAUCHER, Paris, Champion («Champion Classiques Moyen Âge», 17), 2006, pp. 496.

1 Auteur d'une thèse sur la genèse et l'évolution de cette légende du Moyen Âge à nos jours (Robert le Diable. Histoire d'une légende, Paris, Champion, 2003: voir «SF», 144, p. 580 ), Élisabeth Gaucher foumit ici l'édition bilingue du roman anonyme du XIII ${ }^{\mathrm{e}}$ siècle qui se situe au début de la tradition du texte en langue française.

Des origines diaboliques en Normandie à l'expiation de la faute à Rome et jusqu'à la sainteté, Robert est le protagoniste d'un roman qui puise des motifs hérités d'un riche réservoir, comme le montre l'étude préliminaire à cette édition: motifs folkloriques (pacte avec le diable, hybridité de l'enfant, folie démoniaque), épiques (démesure du protagoniste, croisade contre les Turcs), hagiographiques (folie mystique et pénitentielle, interventions du Ciel et communication avec l'au-delà), merveilleux féerique et christianisé (rencontre avec l'ange), se fondent agréablement dans un récit qui trace un parcours ascensionnel de l'enfer - péché de la mère et actes diaboliques de l'enfant - au paradis, avec la sainteté gagnée par Robert. Il paraît par conséquent superflu de s'interroger, mais Élisabeth Gaucher le fait avec un grand équilibre et dirait-on - sans trop y croire elle-même (cf. pp. 32-33), sur l'ancrage historique du 
roman et sur le modèle du protagoniste, que certains critiques, surtout au XIX ${ }^{\mathrm{e}}$ siècle, ont voulu reconnaître dans l'un ou l'autre des ducs de Normandie aux XI ${ }^{\mathrm{e}}$ et XII ${ }^{\mathrm{e}}$ siècles.

3 Transmis par deux manuscrits conservés à la $\mathrm{BnF}$, Robert le Diable, roman d'une longueur moyenne (moins de 5000 vers selon le ms. $B$, un peu plus selon $A$ ), a déjà eu droit à deux éditions: la première, par Guillaume Stanislas Trébutien en 1837, n'était qu'un fac-simile du ms. A (édition rarissime, tirée à 130 exemplaires); la seconde, par Eilert Löseth (SATF, 1903), est une véritable édition critique, fondée sur A. Élisabeth Gaucher a pris le parti d'éditer le texte de $B$, postérieur (fin XIVe-début XV $V^{e}$ siècle) moins fautif mais lacuneux en raison de la perte de deux feuillets: les deux fragments manquants, vv. 1731-1898 et 2251- 2480, sont ici remplacés par les sections correspondantes de $A$, malgré l'écart entre les deux textes. L'examen des variantes textuelles ( $B$ présente des suppressions, des réductions, des ajouts par rapport à $A$ ) prouve bien que le copiste-auteur de ce manuscrit focalisait son attention bien plus sur la dimension individuelle des personnages que sur les faits chevaleresques.

Cette nouvelle édition est précédée d'une étude linguistique détaillée, qui examine tous les aspects essentiels de la copie: graphie, phonétique, morphologie, syntaxe, versification. La note sur la traduction (moins de trois lignes, p. 86) ne rend vraiment compte ni des principes qui ont présidé au travail d'Élisabeth Gaucher (on ne saurait qualifier sa traduction de «la plus fidèle possible au texte original») ni de sa qualité: de fait, l'aisance et la simplicité apparente de la version en français moderne ne peuvent que frapper le lecteur.

5 J'ajouterai quelques observations sur des locutions, images, proverbes, qui ne sont pas tous commentés dans les notes au texte, et dont quelques-uns ne rentrent pas dans le bagage des formules communes et usées de l'écriture narrative du XIII siècle. Parmi les expressions imagées, j'aurais signalé aussi: «Nulz fors tempiés, tant soit bruians, | $\mathrm{Ne}$ vint de si tresgrant aï» (vv. 2558-59) pour souligner l'ardeur avec laquelle Robert s'élance contre les Turcs; l'image qui souligne la blancheur de l'armure du sénéchal est certainement plus banale: «Mais al chief a le coiffe blance | Plus que n'est noif qui est sor brance» (vv. 3887-88); quant à: «Onques [l'empereur] n'en retint en sa part | Qui vaulsist .i. oef de geline», v. 3247, on verra le répertoire de Frankwalt Möhren (Le renforcement affectif de la négation par l'expression d'une valeur minimale en ancien français, Tübingen, Niemeyer, 1980) s.v. uef, p. 232, avec renvoi à notre texte. Quelques comparisons animalières valent aussi la citation; les deux qui suivent, rapportées à Robert, ne sont pas commentées dans l'édition: «Espreviers, quant il vole a quaille, | $\mathrm{Ne}$ vole pas plus de ravine | Que il vers la gent sarasine | Ne voist plus durement assés» (vv. 1924-27: cf. Di Stefano, Dictionnaire des locutions en moyen français, Montréal, CERES, 1991, s.v. épervier); «Onques lyons ne leus warous | Ne fist si grant essil de proie | Com Robiers fist a celle voie» (vv. 3044-46; le commentaire, p. 263, n. 118, ne porte que sur le motif du loup-garou dans la litt. méd.). Pour les proverbes et sentences, il aurait fallu, me semble-t-il, au moins signaler: «Et boine amour toute rien vaint» (v. 3338, à rapporter à la forme ovidienne, très répandue dans la narrative médiévale, «Amor vincit omnia»; cf., outre Morawski, n. 89, Élisabeth Schulze-Busacker, Proverbes et expressions proverbiales dans la littérature narrative du Moyen Âge français. Recueil et analyse, Paris, H. Champion, 1985, pp. 180-181); ainsi que «Chou c'om piert ne puet on garder» (v. 2662, cf. «TPMA», s.v. verlieren, vol. 12, p. 185, n. 63, avec renvoi à ce même vers).

6 Les principes d'édition et de traitement du texte sont soigneusement indiqués (pp. 45-49), bien que certaines appréciations nous paraissent discutables; ainsi lorsque l'on 
attribue au copiste des 'fautes' d'inattention, alors que les graphies auxquelles on fait allusion sont absolument interchangeables au XIII ${ }^{\mathrm{e}}$ siècle (p. 46): «le copiste ne distingue pas toujours $i$ et $j, u$ et $v »$ ou «le copiste confond parfois $c$ et $t$ (les exemples à l'appui sont les graphies adont pour adonc, dont pour donc).

7 L'édition est dans l'ensemble conservative, les corrections s'appuyant sur A (cf. pp. 48-49). Le texte est correct, et la traduction - comme on l'a déjà relevé - se signale par son élégance; l'acception de «tournoi» au v. 1958 («Sor .i. autre tornoi s'eslaisse», traduit par «[Et Robert de s'élancer] vers un autre corps de bataille») n'est enregistrée ni dans Gdf. ni dans FEW: il faudrait sans doute interpréter plus simplement «vers un autre combat» (FEW 13/2, 61b, s.v. tornare). Quelques remarques sur le texte: aux vv. 379-80 la ponctuation est à rectifier: "Quant les messages eut ö̈, / Robers forment s'en esjoÿ» (ce qui correspond à la traduction: «En entendant les messagers, Robert fut rempli de joie»); l'erreur manifeste des vv. 400-401, par ailleurs commentée dans la n. 42 p. 111, n'est pas corrigée: «Mais quant vint a la Penthecouste, | Li rois (à lire: $L i ~ d u c)$ si riche court adjouste; | Ains telz ne fu par duc (à lire sans doute: par roi) tenue». Au v. 1453 la graphie «a ssejour» («A Robiert vint moult grant desir | De dormir assés a ssejour») ne me paraît pas fautive, ni déterminée par la proximité de l'adverbe "assés» (cf. la n. 91, p. 171; pour ces graphies cf. la mise au point de Philippe Ménard, citée ici dans la Bibliographie, p. 492, «Problèmes de paléographie et de philologie dans l'édition des textes français du Moyen Âge», in The Editor and the Text, Edinburgh University Press, 1990, pp. 1-10, en particulier p. 8). Au v. 1543, lire «L'une (et non pas L'un) heure court, l'autre sautielle»; à peine plus loin, v. 1551, plutôt que d'intégrer un deuxième $l$ («il [l']ont»), je lirai: «Et quand i l'ont tant demené». On enregistre quelques coquilles dans la traduction («aux premiers rangs» au pluriel, p. 197; «ils avaient été laissés là», p. 265) et dans les renvois bibliographiques en note (p. 89, n. 1: l'éditeur italien s'appelle «Mucchi» et non pas «Muechi»; Philippe de Novarre, p. 109, n. 39, devrait perdre un -r).

Les variantes du ms. $A$ et les corrections introduites par Löseth dans son édition sont réunies aux pp. 357-386. Une annexe à part est consacrée aux passages de $A$ présentant un texte trop different de celui de B pour être classés parmi les variantes (pp. 387-418). Suivent: l'Index des noms propres (pp. 419-20) et un important Glossaire (pp. 421-485): si les principes d'organisation et de présentation des lemmes sont bien indiqués, aucune référence n'est faite aux dictionnaires utilisés, pour lesquels on se rapportera à la bibliographie, p. 494. Celle-ci est organisée en sections: Éditions et traductions, Études littéraires, Études historiques (sur les prototypes de Robert le Diable), Études philologiques (à la p. 492, le titre de l'article de Paul Meyer est à cor- riger: il ne s'agit pas de «Introduction pour la publication des textes en a. fr.», mais de "Instruction...»; dans le tout dernier paragraphe, consacré à la lexicographie, locutions, proverbes, on est frappé par la collocation de Di Stefano à la lettre «S», et par le renvoi à la 'petite' édition de son dictionnaire [Toutes les herbes de la Saint-Jean, Montréal, CERES, 1992] plutôt qu'au gros répertoire de l'année précédente, qui n'a pas de sous-titre; ni le FEW, ni le répertoire de Schulze Busacker, ni le TPMA ne sont enregistrés). D'autre part, pour les références citées dans les notes de l'introduction, Élisabeth Gaucher garde la fâcheuse habitude d'indiquer la date d'impression des ouvrages plutôt que celle de la première édition: ainsi l'éd. Micha du Merlin de Robert de Boron (1979) est datée ici 2000 (n. 3 p. 14), l'éd. M. Roques du Chevalier au Lion (1960) 1982 (n. 13 p. 18), l'éd. du même M. Roques du Chevalier de la Charrete (1958) 1983 (n. 14 p. 19). 
9 Au-delà des qualités de la traduction, qui donnera accès à ce beau roman du XIII ${ }^{\mathrm{e}}$ siècle à un public plus vaste que le petit cercle des médiévistes, on reconnaîtra à l'édition d'Élisabeth Gaucher le mérite d'avoir mis à la disposition des spécialistes le deuxième manuscrit de Robert le Diable, qui représente déjà, et par sa date et par l'état du texte qu'il transmet, une première réception / adaptation de l'«original». 